N. Oka

Nagoya Math. J.

Vol. 117 (1990), 37-61

\title{
NOTES ON LYAPUNOV GRAPHS AND NON-SINGULAR SMALE FLOWS ON THREE MANIFOLDS
}

\author{
NOBUATSU OKA
}

\section{§1. Introduction}

In the 1980s, Franks, Pugh and Shub raised the question "Given any subshift of finite type $\sigma_{A}: \Sigma_{A} \rightarrow \Sigma_{A}$ is there a non-singular Smale flow (or an NS flow for short) on $S^{3}$ with the suspension of $\sigma_{A}$ as a basic set?" (See [5] and [12]).

In 1985, Franks introduced the concept of a Lyapunov graph, and using this graph, he obtained an affirmative answer about the question (see [6]). Simultaneously, he characterized the Lyapunov graphs appearing as that of NS flows on $S^{3}$. (See Theorem 1 [6]). On the other hand, J. Birman, R.F. Williams studied what kinds of knots appear as sets of closed orbits of knot holders of an NS flow on $S^{3}$. (See [2]). For nonsingular Morse Smale flows (or NMS flows for short), which are special cases of NS flows, Sasano and Wada characterized the knot types or link types which appear as sets of closed orbits. Kobayashi also studied the types of primitive links in the case of special Seifert manifolds. (See [10], [14] and [16]).

Our purpose of this notes is to decide what kinds of Lyapunov graphs appear on certain three manifolds associated with NS flows. By using this graph, the global conditions of flows on manifolds are more visible than other methods.

Our first result in Theorem A is a characterization of the Lyapunov graphs of NS flows on $L(2 p-1, q)$ by using a standard technique of 3dimensional topology, which extends Theorem 1 of Franks' paper [6]. For NMS flows, Franks characterized the type of Lyapunov graphs assciated with NMS flows on $S^{3}$. (See Theorem 2 [6]). In our notes, we define a notion of a singular vertex for Lyapunov graphs associated with NMS flows on the irreducible, orientable, closed 3-manifolds which can

Received March 4, 1988. 
not admit incompressible tori. By observing that the Lyapunov graphs on the case of the above manifolds which are not lens space must have one singular vertex in Theorem $B$, we extend Theorem 2 [6] to the case of the irreducible, orientable, closed 3-manifolds which can not admit incompressible tori. Moreover, we define a special Lyapunov graph which distinguishes a singular vertex from an ordinary vertex. We characterize the types of the special graphs on the case of lens space. (See Proposition 5 and Proposition 6).

I would like to express my gratitude to professors K. Shiraiwa, G. Ikegami, and S. Negami for their useful advice and encouragement.

\section{§2. Definitions and preliminaries}

For the definition of an irreducible 3-manifold, a Seifert fibered space, an incompressible surface, etc., we refer to Hempel [7] and Jaco [8].

Definition 1. Suppose that $\phi_{t}: M \rightarrow M$ is a continuous flow and $\varepsilon>0$, we say there is an $\varepsilon$-chain from $x$ to $y$ provided that there exist points $x_{1}=x, x_{2}, \cdots, x_{n+1}=y$ and real numbers $t(i)>1$ such that $d\left(\phi_{t(i)}\left(x_{i}\right)\right.$, $\left.x_{i+1}\right)<\varepsilon$ for all $1 \leqq i \leqq n$. A point $x$ is called chain recurrent if for any $\varepsilon>0$ there is an $\varepsilon$-chain from $x$ to $x$. The set $R$ of all chain recurrent points is called the chain recurrent set.

Definition 2. If $\phi_{t}: M \rightarrow M$ is a smooth flow, then a smooth function $g: M \rightarrow \mathbb{R}$ will be called a Lyapunov function provided

(1) $d\left(g\left(\phi_{t}(x)\right) / d t<0\right.$ if $x \in R$ and

(2) when $x, y \in R, g(x)=g(y)$ if and only if for each $\varepsilon>0$ there are $\varepsilon$-chains from $x$ to $y$ and $y$ to $x$.

Definition 3. A smooth flow $\phi_{t}: M \rightarrow M$ on a compact manifold is called a Smale flow provided that

(1) its chain recurrent set $R$ has a hyperbolic structure, $\operatorname{dim} R \leqq 1$ and that

(2) it satisfies the transversality condition.

Remark 1. When a Smale flow does not have any singular points, it is called a non-singular Smale flow (or an NS flow for short).

Remark 2. A flow $\phi_{t}: M \rightarrow M$ is especially called an NMS flow when its chain recurrent set $R$ consists of finitely many hyperbolic closed orbits and satisfies the transversality condition. 
Definition 4. An (abstract) Lyapunov graph is a finite connected oriented graph which possesses no oriented cycles, and each vertex of which is labelled with a chain recurrent flow on a compact space.

From now on we assume that $M$ is an irreducible, orientable, closed 3-manifold. Since we study an NS flow on $M$, each vertex of a Lyapunov graph will be labelled with a basic set, which in this case is topologically equivalent to either a suspension of a subshift of finite type $\sigma_{A}$ : $\Sigma \rightarrow \Sigma$ corresponding to a matrix $A$ (see Bowen [1]), an attracting closed orbit, a repelling closed orbit, a closed orbit of twisted saddle type, or a closed orbit of untwisted saddle type.

We note that all attractor and repeller of an NS flow are closed orbit; because a hyperbolic chain recurrent set satisfies Axiom A (see Franke and Selgrade [3], and Smale [15]), each basic set has a closed orbit. If a basic set $\Lambda$ is attracting or repelling and $\operatorname{dim} \Lambda=1, \Lambda$ must be an isolated orbit.

Definition 5. Suppose $\phi_{t}$ is a Smale flow on $M, g: M \rightarrow \mathbb{R}$ is a Lyapunov function and $\Lambda$ is a basic set with $g(\Lambda)=C$. We will say that $X=g^{-1}([C-\varepsilon, C+\varepsilon])$ is a basic block for $\Lambda$, if the following three conditions are satisfied.

(1) $X$ contains only one basic set.

(2) There exist (not necessarily connected) codimension one submanifolds $U$ and $V$ with boundary in $X$ such that $U \subset V$ and that they are transverse to the flow.

(3) Let $H$ be a finite set of one handles $h_{i}\left(\cong D^{1} \times D^{1}\right)$ in $U$; $H=$ $\cup_{i} h_{i}$ the first return map $r: U \rightarrow$ int $V$ is well defined, smooth and there is a hyperbolic handle set $H \subset$ int $U$, with every orbit of $\Lambda$ intersecting $H$ and $h_{i} \subset H$ intersecting $\Lambda$. Here we call a handle set $H$ hyperbolic handle, if it satisfies the followings.

(1) If $x \in h_{i}$ and $r(x) \in h_{j}$, then $\operatorname{int}\left(r\left(W_{i}^{u}(x)\right) \supset W_{j}^{u}(r(x))\right.$ and $r\left(W_{i}^{s}(x)\right)$ $\subset \operatorname{int}\left(W_{j}^{s}(r(x))\right.$.

(2) There is a $\lambda \in(0,1)$ such that for each $x \in h_{i}$, with $r(x) \in H$ and each $v \in T_{x}\left(W_{i}^{s}(x)\right), w \in T_{x}\left(W_{i}^{u}(x)\right)$ we have $\|d r(v)\| \leqq \lambda\|v\|,\|d r(w)\| \geqq \lambda^{-1}\|w\|$. Here $W_{i}^{u}(x)$ denotes the interval $D^{1} \times\{p\} \subset h_{i}$ which contains a point $x$. Similarly $W_{i}^{s}(x)$ denotes the interval $\{q\} \times D^{1} \subset h_{i}$ containing $x$.

Definition 6. Suppose $\Lambda_{j}$ is a basic set of a Smale flow contained in a basic block $X_{j}$ with hyperbolic handles $H$, then we call $K\left(\Lambda_{j}\right)=$ 
$\bigcup_{t \in \mathrm{R}} \phi_{t}(H)$ the saturated handles. $K\left(\Lambda_{j}\right)$ has stable foliations $W^{s}(x)$ with leaves containing $x \in K$, and $y \in W^{s}(x)$ if and only if there exists $t_{1}, t_{2} \in R$ and $z \in h_{i} \subset H$ such that $\phi_{t 1}(x), \phi_{t 2}(y)$ are both in $W_{i}^{s}(z) \subset h_{i}$. The unstable leaf $W^{u}(x)$ has a similar property.

Definition 7. A Smale flow $\phi_{t}$ on $M$ will be called fitted provided

(1) there exists a Lyapunov function $g: M \rightarrow \mathbb{R}$ with respect to which a basic set $\Lambda_{j}$ has a basic block $X_{j}$ and $\left\{X_{j}\right\}$ are pairwise disjoint, and

(2) if $x \in K\left(\Lambda_{i}\right), y \in K\left(\Lambda_{j}\right)$ and $g(x)>g(y)$, then $W^{u}(x)$ either contains $W^{u}(y)$ or is disjoint from it and $W^{s}(y)$ either contains $W^{s}(x)$ or is disjoint from it.

Remark 3. A fitted Smale flow satisfies the transversality conditions.

Lemma 1. Suppose that $\phi_{t}: M \rightarrow M$ is a non-singular smooth flow on an irreducible, orientable, closed 3-manifold $M$, and suppose that $g$ : $M \rightarrow \mathbb{R}$ is a Lyapunov function associated with a flow $\phi_{t}$. Then each component of a level surface of a regular value of the Lyapunov function $g$ is homeomorphic to a torus.

Proof. Suppose that we cut manifold $M$ at the level $g^{-1}(C)$, where $C$ is a regular value of the Lyapunov function $g$. Let $g^{-1}([C,+\infty))$ be denoted by $M^{+}$. Suppose that a flow exits transversely on the boundary $\partial M^{+}$. Thus considering a double of $M^{+}$, we obtain $\chi\left(\partial M^{+}\right)=0$, where $\chi\left(\partial M^{+}\right)$denotes the Euler characteristic of $\partial M^{+}$. If there is a surface of genus greater than or equal to two in $g^{-1}(C)=\partial M^{+}$, the boundary of $g^{-1}(C)$ must contain $S^{2}$. Since $M$ is irreducible, the $S^{2}$ bounds a ball in $M$. But there exists a singular point in the ball. This is a contradiction. Then each component of $\partial M^{+}$is a torus.

LEMMA 2. Let $T^{2}$ be a compressible torus in an irreducible, orientable, closed 3-manifold $M$. Then $T^{2}$ bounds a solid torus or there exists a nontrivial knot $h$ in $S^{3}$ such that $T^{2}$ bounds a compact 3-manifold $M$ which is homeomorphic to $S^{3}-N(h)$, where $N(h)$ denotes a regular neighbourhood of $h$. Moreover, in the last case we can take a boundary of a compressing disk of $T^{2}$ for a meridian of $h$.

Proof. Let $T^{2}$ be a compressible torus in $M$. Then there exists an essential simple closed curve on $T^{2}$ which bounds a disk $D^{2}$ in $M$. By Dehn's Lemma, $D^{2}$ is a non-singular disk. Thus the $\left.\left(T^{2} \cap \overline{\left(M-\left(D^{2} \times I\right)\right.}\right)\right)$ $\cup D^{2} \times \partial I$ is homeomorphic to $S^{2}$. The $S^{2}$ bounds a ball in $M$. Here $K$ 
denotes a closure of an outside of the torus as in Figure (1). Let $\overline{M-\left(K \cup D^{2} \times I\right)}$ be denoted by $A$. Then if the ball $B^{3}$ corresponds to $A$, we see that $A \cup D^{2} \times I$ is homeomorphic to a solid torus. It means that the $T^{2}$ bounds a solid torus in $M$. If the ball $\mathrm{B}^{3}$ corresponds to $K \cup D^{2} \times I$, we see that $T^{2}$ bounds $K=B^{3}-D^{2} \times I$. Thus when $K$ is embedded in $S^{3}$, it may be a non-trivial knot complement $S^{3}-N(h)$. Moreover, in this case, the boundary of a compressible disk of the $T^{2}$ can be taken as the boundary of $D^{2} \times\{p\}$ of the $D^{2} \times I$ in $M$. Then we see that the disk $D^{2} \times\{p\}$ is a meridian disk of $h$ in $S^{3}$.

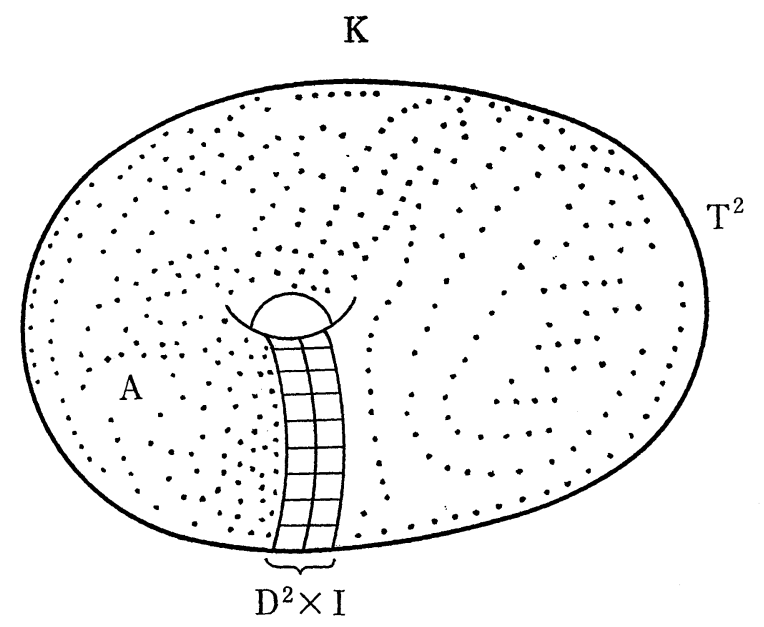

Figure (1)

Proposition 1. Suppose that $M$ is an orientable, irreducible, closed 3-manifold, and that $M$ does not admit incompressible tori. Let $\phi_{t}: M \rightarrow$ $M$ be a non-singular smooth flow with a Lyapunov function $g: M \rightarrow \mathbb{R}$. Let $\Gamma$ be a Lyapunov graph associated with the flow $\phi_{t}$ and Lyapunov function $g$. Then $\Gamma$ is a tree.

Proof. By Lemma 1, each component of $g^{-1}(C)$ is a torus, where $C$ denotes a regular value of $g$. Since $M$ is an irreducible manifold and since $M$ does not have any incompressible torus, each component of $g^{-1}(C)$ bounds a solid torus or a non-trivial knot complement by Lemma 2. Hence $\Gamma$ is a tree.

Remark 4. If $H_{1}(M, \mathbb{Q})=\{0\}$ for a 3-manifold $M$, a Lyapunov graph $\Gamma$ associated with a flow $\phi_{t}: M \rightarrow M$ and a Lyapunov function $g: M \rightarrow \mathbb{R}$ 
is a tree. In this case, the flow $\phi_{t}$ is not necessarily non-singular (see [6]).

Remark 5. There exists an irreducible, orientable, closed 3-manifold $M$ which does not admit incompressible tori and $H_{1}(M, \mathbb{Q}) \neq\{0\}$ in the category of Seifert manifolds whose orbit spaces are $S^{2}$ with three exceptional fibers (See [8]).

Proposition 2 ([6]). Let $X_{i}(i=1,2, \cdots, n-1)$ be a 3-dimensional basic block in a 3-manifold $M$. Suppose that $h_{i}: \partial X_{i}^{-} \rightarrow \partial X_{i+1}^{+}$is a diffeomorphism $(1 \leqq i \leqq n-1)$, where $\partial X_{i}^{-}$is the part of the boundary $\partial X_{i}$ on which the flow exits, and $\partial X_{i+1}^{+}$is the part of the boundary $\partial X_{i+1}$ on which the flow is entering $(1 \leqq i \leqq n-1)$. For each $i, h_{i}$ is isotopic to $g_{i}: \partial X_{i}^{-} \rightarrow$ $\partial X_{i+1}^{+}$such that the flow on $X_{1} \cup_{g_{1}} X_{2} \cup_{g_{2}} \cdots \cup_{g_{n-1}} X_{n}$ is fitted Smale flow.

\section{§3. Main results}

The main results of this paper is as follows.

(1) Case of Smale flow

TheOREM A. Let $\phi_{t}$ be an NS flow on a lens space $L(2 p-1, q)$ and let $g$ be a Lyapunov function. Then a Lyapunov graph $\Gamma$ associated with the flow $\phi_{t}$ and the function $g$ satisfies the following conditions (0), (1) and (2).

(0) It is a tree with one edge attached to each source and sink vertex.

(1) Each source (resp. each sink) vertex is labelled with an attracting closed orbit (resp. a repelling closed orbit).

(2) If $V$ is any other vertex, it is labelled with a suspension of the subshift of finite type $\sigma(A)$ with a transition matrix $A$ which is an irreducible $n \times n$ matrix. Let $e_{V}^{+}$be the number of entering edges and let $e_{V}^{-}$be the number of exiting edges. If $k_{V}=\operatorname{dim} \operatorname{ker}\left(\left(I-\bar{A}_{V}\right): Z_{2}^{n} \rightarrow Z_{2}^{n}\right)$, where $\bar{A}_{V}$ is the $\bmod 2$ reduction of $A_{V}$, then $e_{V}^{+} \leqq k_{V}+1, e_{V}^{-} \leqq k_{V}+1$ and $k_{V}+1$ $\leqq e_{V}^{+}+e_{V}^{-}$.

Conversely, if an abstract Lyapunov graph $\Gamma$ satisfies the above conditions (0), (1) and (2), then there exists an NS flow on $L(2 p-1, q)$ whose Lyapunov graph is $\Gamma$.

(2) Case of Morse-Smale flow

Definition 8. Suppose that $\Gamma$ is a Lyapunov graph associated with an NS flow $\phi_{t}$ and a Lyapunov function $g$ on an irreducible, orientable, 
closed 3-manifold $M$ which does not admit incompressible tori. If there is a vertex of $\Gamma$ which satisfies following conditions, we call the vertex a singular vertex.

(1) If we cut the graph $\Gamma$ at any point $C^{+}$of any entering edge of a vertex $V$, a submanifold of $M$ which corresponds to a component of $\Gamma$ containing the vertex $V$ is neither a soild torus nor a non-trivial knot complement $S^{3}-N(h)$ such that the boundary of a compressing disk of $\partial\left(S^{3}-N(h)\right)$ is a meridian of this knot $h$.

(2) If we cut the graph $\Gamma$ at any point $C^{-}$of any exiting edge of the above vertex $V$, a submanifold of $M$ which corresponds to a component of $\Gamma$ containing the vertex $V$ is also neither a solid torus nor a non-trivial knot complement which satisfies the condition of the case (1).

Definition 9. If a vertex $V$ corresponds the closed orbit of twisted saddle type, then we call the vertex $V$ a twisted vertex, An untwisted vertex is defined similarly. Here a saddle type closed orbit is called untwisted or twisted if the associated unstable bandle $E^{U}$ is oreintable or not.

TheOREm B. Suppose that $\phi_{t}$ is an NMS flow on an irreducible, orientable, close 3-manifold $M$ which does not admit incompressible tori and suppose that $g$ is a Lyapunov function. Then a Lyapunov graph $\Gamma$ associated with the flow $\phi_{t}$ and the function $g$ satisfies the followings.

(a) Case of $M$ being a lens space:

(1) It is a tree with one edge attached to each source or sink vertex.

(2) If $V$ is a vertex labelled with the closed orbit of saddle type and the vertex $V$ has $e_{V}^{+}$enterning edges, and $e_{V}^{-}$exiting edges, then $e_{V}^{+}=1$ or $2, e_{V}^{-}=1$ or 2 and if $V$ is twisted, then $e_{V}^{+}=e_{V}^{-}=1$.

(b) Case of $M$ not being a lens space:

(b1) Suppose $M$ admits a Seifert fibration whose orbit manifold is $S^{2}$ with three exceptional fibers. And suppose that it does not admit a Seifert fibration whose orbit space is a projective plane $P^{2}$.

(1) The graph is a tree with one edge attached to each source or sink vertex.

(2) There exists one (singular) vertex $V^{*}$ labelled with a closed orbit of saddle type, and suppose $V^{*}$ has $e_{V^{*}}^{+}$entering edges and $e_{V^{*}}^{-}$ exiting edges. If $V^{*}$ is untwisted then either $e_{V^{*}}^{+}=2$ and $e_{V^{*}}^{-}=1$, or $e_{V^{*}}^{+}=1$ and $e_{V^{*}}^{-}=2$. If $V^{*}$ is twisted, then $e_{V^{*}}^{+}=e_{V^{*}}^{-}=1$. 
Suppose $V$ is any other vertex labelled with a saddle orbit, and has $e_{V}^{+}$entering edges and $e_{\vec{V}}^{-}$exiting edges. If $V$ is untwisted, then $e_{V}^{+}=2$ or $1, e_{\bar{V}}^{-}=2$ or 1 . If $V$ is twisted, then $e_{V}^{+}=e_{V}^{-}=1$.

(b2) Suppose that $M$ admits a Seifert fibration whose orbit manifold is $S^{2}$ with three exceptional fibers. And suppose that it also admits a Seifert fibration whose orbit manifold is a projective plane $P^{2}$ with at most one exceptional fiber.

(1) The graph is a tree with one edge attached to each source or sink vertex.

(2) There exists one (singular) vertex $V^{*}$ labelled with an orbit of saddle type. Suppose $V^{*}$ has $e_{V^{*}}^{+}$entering edges and $e_{V^{*}}^{-}$exiting edges. If $V^{*}$ is untwisted, then $e_{V^{*}}^{+}=2$ and $e_{V^{*}}^{-}=1, e_{V^{*}}^{+}=1$ and $e_{V^{*}}^{-}=2$, or $e_{V^{*}}^{+}=e_{V^{*}}^{-}=1$. If $V^{*}$ is twisted, then $e_{V^{*}}^{+}=e_{V^{*}}^{-}=1$, Suppose $V$ is any other vertex labelled with an orbit of saddle type and has $e_{V}^{+}$entering edges and $e_{V}^{-}$existing edges. If $V$ is untwisted, then $e_{V}^{+}=1$ or $2, e_{V}^{-}=1$ or 2 and if $V$ is twisted, $e_{V}^{+}=e_{V}^{-}=1$

Conversely, if an abstract Lyapunov graph $\Gamma$ satisfies conditions (1) and (2) of case (a), case (b1), and case (b2) respectively, then there exists an NMS flow $\phi_{t}$ on $M$ whose Lyapunov graph is $\Gamma$.

Remark 6. Kobayashi detects the following. If an irreducible, orientable, closed 3-manifold $M$ which does not admit incompressible tori admits an NMS flow, then $M$ is one of the following manifolds.

(1) lens space (2) Seifert manifold whose orbit space is $S^{2}$ with three exceptional fibers. (See Kobayashi [10] and also see Morgan [11]).

\section{§4. Proof of Theorem A and Theorem B}

We will use the next Lemma and Proposition for the necessary condition in Theorem A and Theorem B.

Lemma 3. Suppose $V$ is a singular vertex of a Lyapunov graph $\Gamma$. If we cut the graph $\Gamma$ at any cut point on its edges, the submanifold of $M$ which corresponds to the component of $\Gamma$ containing the vertex $V$ is neither a solid torus nor a non-trivial knot-complement $S^{3}-N(h)$ such that the boundary of compressing disk of $\partial\left(S^{3}-(N(h))\right.$ is a meridian of this knot $h$. 
Proof. Suppose that we cut the graph $\Gamma$ at a cut point on an edge of a vertex $V$. Let $\Gamma^{\prime}$ denote a component of $\Gamma$ which contains a singular vertex $V$, and let $N$ denote a submanifold of $M$ which corresponds to $\Gamma^{\prime}$. Suppose $N$ is a solid torus or a knot-complement. Attaching one solid torus which contains an attracting closed orbit or a repelling closed orbit along the boundary of $N$, we can construct a non-singular smooth flow on $S^{3}$. Then we can construct a new Lyapunov graph $\Gamma^{\prime \prime}$. By the Solid Torus Theorem (see [13]), any torus in $S^{3}$ bounds a solid torus on one side and bounds a knot-complement which satisfies the condition of Lemma 3 on the other side. Then if we cut the graph $\Gamma^{\prime \prime}$ at any point $C^{*}$ of any entering edge of $V_{0}$ or any exiting edge of $V_{0}$, each component of $\Gamma^{\prime \prime}$ is a solid torus or a knot-complement. This is a contradiction. Thus $N$ is neither a solid torus nor a knot-complement which satisfies the condition of Lemma 3 .

Proposition 3. Suppose that $\phi_{t}$ is a non-singular Smale flow on an irreducible, orientable, closed 3-manifold $M$ which does not admit incompressible tori, and suppose that $\Gamma$ is a graph associated with a flow $\phi_{t}$ and a Lyapunov function $g$, then a vertex of $\Gamma$ which is neither an attracting nor a repelling satisfies the following conditions.

(1) There exists at most one singular vertex $V_{0}$ labelled with the suspension of a subshift $\sigma\left(A_{V_{0}}\right)$. Suppose $V_{0}$ has $e_{V_{0}}^{+}$entering edges and $e_{\bar{V}_{0}}$ exiting edges. If $k_{V}=\operatorname{dim} \operatorname{ker}\left(\left(I-\bar{A}_{V_{0}}\right): \mathbb{Z}_{2}^{n} \rightarrow \mathbb{Z}_{2}^{n}\right)$, where $\bar{A}_{V_{0}}$ is the $\bmod 2$ reduction of $A_{V_{0}} \cdot e_{V_{0}}^{+} \leqq k_{V_{0}}+1, e_{V_{0}}^{-} \leqq k_{V_{0}}+1, k_{V_{0}}+1-\operatorname{dim} H_{1}\left(M, \mathbb{Z}_{2}\right) \leqq$ $e_{V_{0}}^{+}+e_{\bar{V}_{0}}^{-}$.

(2) Any other vertex $V$ satisfies condition (2) in Theorem A. Moreover, if $M$ is not a lens space, $\Gamma$ must have exactly one singular vertex.

Proof. By Proposition 1, the Lyapunov graph $\Gamma$ is a tree. Suppose that there are two singular vertices $V_{1}$ and $V_{2}$ in $\Gamma$. Then $V_{1}$ is contained in a component of $\Gamma-\{C\}$ corresponding to a solid torus or a knot-complement in $M$, where $C$ is a cut point of an entering edge or an exiting edge of the vertex $V_{2}$. Then by Lemma $3, V_{1}$ is not a singular vertex. Thus the number of singular vertices in $\Gamma$ is at most one. Now we suppose that $\Gamma$ has a singular vertex $V^{*}$, and we choose cut points $\left\{C_{1}, \cdots, C_{n}\right\}$ on all entering edges and all exiting edges of singular vertex $V^{*}$. By Lemma 1 and Lemma 2, each component which does not contain the singular vertex $V^{*}$ corresponds a solid torus or a nontrivial knot-complement $S^{3}-N(h)$. Now, we will cut $\Gamma$ at a cut point 
$C_{i} \in\left\{C_{1}, \cdots, C_{n}\right\}$ and let $\Gamma^{\prime}$ be the component of $\Gamma-\left\{C_{i}\right\}$ which does not contain $V^{*}$. Then $\Gamma^{\prime}$ corresponds a solid torus or a non-trivial knotcomplement $S^{3}-N(h)$ in $M$. By attaching a new solid torus which contains an attractive closed orbit or a repelling closed orbit along the boundary of the solid torus or the knot-complement corresponding to $\Gamma^{\prime}$, we construct a new graph $\Gamma^{\prime \prime}$ associated with a new flow on $S^{3}$. Then each vertex of $\Gamma^{\prime \prime}$ satisfies condition (2) in Theorem A by Franks' Theorem 1 in [6]. Thus the vertex which is not a singular vertex satisfies the condition (2) in Theorem A. Let $C$ be a level of the singular vertex $V^{*}$ of $\Gamma$ for the Lyapunov function $g$. By Lemma 2, each component of $\Gamma$ corresponding with $g^{-1}([C+\varepsilon,+\infty))=Y$ is a solid torus or a nontrivial knot-complement, and also $g^{-1}((-\infty, C-\varepsilon])=Z$ is a solid torus, or a knot-complement in $M$. Let $X$ be $g^{-1}((-\infty, C+\varepsilon])$, then $X \cup Y=M$ and $X \cap Y=\partial X$ consists of disjoint $e_{V_{0}}^{+}$tori.

Now, we consider the following Mayer-Vietoris exact sequence with in $\mathbb{Z}_{2}$.

$$
H_{3}(X) \oplus H_{3}(Y) \longrightarrow H_{3}(X \cup Y) \longrightarrow H_{2}(X \cap Y) \stackrel{\alpha_{*}}{\longrightarrow} H_{2}(X) \oplus H_{2}(Y) \stackrel{C_{*}}{\longrightarrow} .
$$

Since each component of $Y$ is a solid torus or a knot-complement, $H_{3}(Y)$ $\cong H_{2}(Y) \cong\{0\}$. And we see that $H_{3}(X \cup Y) \cong H_{3}(M) \cong \mathbb{Z}_{2}, H_{3}(X) \cong\{0\}$, and $H_{2}(X \cap Y)=\oplus_{i=1}^{e_{i=1}^{+}} \mathbb{Z}_{2}$ Hence,

$$
\operatorname{dim} \operatorname{ker} C_{*}=\operatorname{dim} \operatorname{Im} \alpha_{*} \leqq \operatorname{dim} H_{2}(X)
$$

and

$$
\operatorname{dim} \operatorname{Im} \alpha_{*}=e_{V_{0}}^{+}-1 .
$$

Next, we consider the exact sequence of the pair $(X, Z)$.

$$
H_{2}(Z) \longrightarrow H_{2}(X) \stackrel{b_{1 *}}{\longrightarrow} H_{2}(X, Z) \longrightarrow H_{1}(Z) \longrightarrow .
$$

Since $H_{2}(Z)=\{0\}, b_{1 *}$ is injective and $\operatorname{dim} H_{2}(X, Z)=\operatorname{dim} \operatorname{ker}(I-\bar{A})=k_{V_{0}}$ (see Franks [6]). Then

$$
\operatorname{dim} H_{2}(X) \leqq k_{V_{0}} .
$$

We see $e_{V_{0}}^{+} \leqq k_{V_{0}}+1$ by (1), (2) and (3) and $e_{V_{0}}^{\bar{V}_{0}} \leqq k_{V_{0}}+1$ follows from considering the reverse flow.

We will show that $e_{V_{0}}^{+}+e_{V_{0}}^{\bar{V}_{0}} \geqq k_{V_{0}}+1-\operatorname{dim} H_{1}(M)$. We see that

$$
\operatorname{dim} H_{2}\left(X, \mathbb{Z}_{2}\right)=\operatorname{dim} H^{2}\left(X, \mathbb{Z}_{2}\right)
$$


and that

$$
H^{2}(X)=H_{1}(M, M-X)
$$

Now, we consider the following exact sequence.

$$
\tilde{H}_{1}(M-X) \longrightarrow \tilde{H}_{2}(M) \longrightarrow H_{1}(M, M-X) \longrightarrow \tilde{H}_{0}(M-X) \longrightarrow .
$$

Then

$$
\operatorname{dim} H_{1}(M, M-X) \leqq e_{V_{0}}^{+}-1+\operatorname{dim} H_{1}(M) .
$$

We consider the next exact sequence of the pair $(X, Z)$ again.

$$
H_{2}(Z) \longrightarrow H_{2}(X) \stackrel{b_{2 *}}{\longrightarrow} H_{2}(X, Z) \longrightarrow H_{1}(Z) \longrightarrow .
$$

By (4), (5), (6) and the facts that $b_{2 *}$ is injective and that $H_{1}(Z)=\oplus_{i=1}^{e_{\overline{V_{0}}}} \mathbb{Z}_{2}$, we obtain that $k_{V_{0}}+1-\operatorname{dim} H_{1}\left(M, Z_{2}\right) \leqq e_{V_{0}}^{+}+e_{\bar{V}_{0}}^{-}$. If $M$ is not a lens space, an NS flow on $M$ has at least one basic set which is neither an attractor nor a repeller. Then an associated Lyapunov graph $\Gamma$ has at least one vertex $V_{0}$ which is neither an attractor nor a repeller. Suppose that an associated graph $\Gamma$ has no singular vertex. Then for any vertex $V_{0}$ which is neither an attractor nor a repeller, there exists a cut point $C_{0}$ on an entering edge $V_{0} V_{1}$ or an exiting edge $V_{0} V_{1}$. The component $\Gamma_{0}$ of $\Gamma-\left\{C_{0}\right\}$ which contains $V_{0}$ corresponds to a submanifold which is homeomorphic to a solid torus or a non-trivial knot-complement. Similarly, there is a cut point $C_{1}$ on an entering edge $V_{1} V_{2}$ or an exiting edge $V_{1} V_{2}$ such that the component of $\Gamma_{1}$ of $\Gamma-\left\{C_{1}\right\}$ which contains $V_{1}$ corresponds to a submanifold which is homeomorphic to a solid torus or a non-trivial knot-complement. Here, if $V_{0}=V_{2}$, the manifold $M$ is homeomorphic to one of (solid torus) $\cup_{T}$ (solid torus), (solid torus) $\cup_{T}$ (non-trivial knot-complement) and (non-trivial knot-complement) $\cup_{T}$ (nontrivial knot-complement), where $A \cup_{T} B$ means a manifold obtained from $A$ and $B$ by identifying $\partial A$ and $\partial B$ which are homeomorphic to a torus $T$. In the first case, $M$ is homeomorphic to a lens space, in the second case, $M$ is homeomorphic to $S^{3}$ by Lemma 2 and in the last case, $M$ has an incompressible torus. Then this is a contradiction. Thus $V_{0} \neq V_{2}$. Then, there is a cut point $C_{2}$ on an entering edge $V_{2} V_{3}$ or an exiting edge $V_{2} V_{3}$ such that the component $\Gamma_{2}$ of $\Gamma-\left\{C_{2}\right\}$ which contains $V_{3}$ corresponds to a submanifold which is homeomorphic to a solid torus or a knot complement. We see $V_{3} \neq V_{1}$ by the above argument. Since $\Gamma$ 
is a tree, $V_{3} \neq V_{0}$. This procedure can be continued indefinitely. But the Lyapunov graph has only finite vertices. This is a contradiction. Thus the Lyapunov graph has exactly one singular vertex.

\section{Proof of Theorem A.}

Necessity. By Proposition 3, all vertices (which are neither an attractor nor a repeller) satisfy $e_{V}^{+} \leqq k_{V}+1, e_{V}^{-} \leqq k_{V}+1$ and $k_{V}+1-$ $\operatorname{dim} H_{1}\left(M, \mathbb{Z}_{2}\right) \leqq e_{V}^{+}+e_{\bar{V}}^{-}$. We note that $H_{1}\left(L(2 p-1, q), \mathbb{Z}_{2}\right)=\{0\}$. Then, it follows that $k_{V}+1 \leqq e_{V}^{+}+e_{\bar{V}}^{-}$. Thus the condition (2) holds. And the condition (0) and (1) are clearly satisfied.

Sufficiency. Let $X_{1}, X_{2}, \cdots, X_{n}$ be basic blocks corresponding with vertices of a given abstract Lyapunov graph satisfying the conditions as in Theorem 1 [6], where $X_{n}$ is especially a basic block corresponding with an attractive closed orbit. Since all boundaries of basic blocks $X_{1}$, $X_{2}, \cdots, X_{n-1}$ are standard tori. By the Solid Torus Theorem [13], we can construct unknotted solid torus $X_{1} \cup_{g_{1}} X_{2} \cup_{g_{2}} \cdots \cup_{g_{n-2}} X_{n-1}$ in $S^{3}$, where $g_{1}, g_{2}, \cdots, g_{n-2}$ are attaching diffeomorphisms. Because $X_{n}$ is a basic block for an attractive closed orbit, it is a solid torus. Then, we can choose an attaching diffeomorphism $g_{n-1}$ such that $\left(X_{1} \cup_{g_{1}} X_{2} \cup_{g_{2}} \cdots \cup_{g_{n-2}} X_{n-1}\right)$ $\cup_{g_{n-1}} X_{n}$ is a lens space $L(2 p-1, q)$. By Proposition 2, we can construct a fitted Smale flow on $L(2 p-1, q)$.

Remark 7. Since the diffeomorphism $h_{i}$ in Proposition 2 is isotopic to $g_{i}$, the type of lens space $L(2 p-1, q)$ is unchanged.

Question. For a singular vertex $V_{0}$, is there a basic block such that $k_{V_{0}}=e_{V_{0}}^{+}+e_{\bar{V}_{0}}^{\bar{N}_{0}}, e_{V_{0}}^{+} \leqq k_{V_{0}}+1$ and $e_{\bar{V}_{0}} \leqq k_{V_{0}}+1$ in lens space $L(2 p, q)$ ?

Concerning the above question, we can construct a basic block such that $k_{V_{0}}=e_{V_{0}}^{+}+e_{V_{0}}^{-}, e_{V_{0}}^{+} \leqq k_{V_{0}}+1$, and $e_{V_{0}}^{\bar{y}_{0}} \leqq k_{V_{0}}+1$ in 3 -torus. But a 3 -torus is not a lens space and it has an incompressible torus.

Construction of a basic block in a 3-torus.

We consider a cross section of $\phi_{t}$, whose first return map has two saddle points and one attractive fixed point as Figure (2). We next add $k_{V}-\left(e_{V}^{+}+1\right)$ pairs of a source and a saddle point on this cross section and also add $k_{V}-\left(e_{\vec{V}}+1\right)$ pairs of a sink and a saddle. Finally we add $\left(n-k_{V}\right)$ nilpotent handles but we do no further isotopy and which contain no chain recurrent points at this moment. (See Theorem 1 in [6]). 


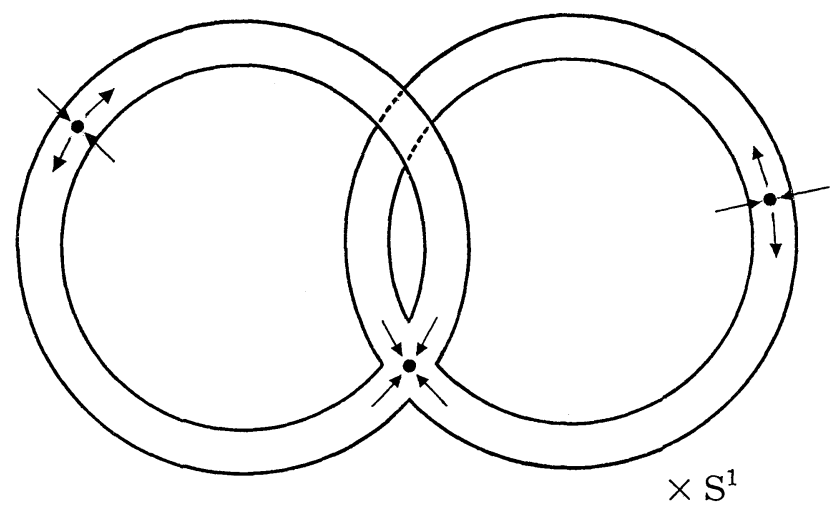

Figure (2)

Then, we can construct basic block such that $k_{V}=e_{V}^{+}+e_{V}^{-}, e_{V}^{+} \leqq k_{V}+1$ and $e_{V}^{-} \leqq k_{V}+1$. By attaching a standard solid torus containing one repelling closed orbit along the boundary of this basic block we obtain a 3 -torus and an NS flow. (Here, $k_{V} \geqq 2$ ).

Remark 8. A closed orbit of saddle type has two dimensional unstable manifold and also two dimensional stable manifold and satisfies $k_{V}=1$. Therefore, there is no saddle type vertex which satisfies $k_{V}=$ $e_{V}^{+}+e_{V}^{-}$in an NMS flow on any 3-manifold.

To prove Theorem B, we need the following proposition.

Proposition 4. Suppose that $M$ is an irreducible, orientable, closed 3-manifold which does not admit incompressible tori, and suppose that $M$ is not a lens space. Let $\phi_{t}$ be an NMS flow on $M$ with a Lyapunov function $g$. Then a Lyapunov graph associated with the flow $\phi_{t}$ and the function $g$ satisfies one of (1), (2) and (3).

(1) (a) $M$ admits a structure of a Seifert fibered manifold whose orbit space is a projective plane $P^{2}$ with at most one exceptional fiber, and (b) a graph $\Gamma$ has one singular vertex $V_{0}$ such that $e_{V_{0}}^{+}=e_{V_{0}}^{-}=1$ and $V_{0}$ is untwisted. Any other saddle type vertex is of $S^{3}$-type, where a vertex of $S^{3}$ type is a vertex which is not a singular vertex, and which satisfies condition. (2) of the case of a lens space in Theorem B.

(2) (a) $M$ admits a Seifert fibration whose orbit manifold is $S^{2}$ with three exceptional fibers and (b) the graph $\Gamma$ has one singular vertex $V_{0}$ such that $e_{V_{0}}^{+}=2$ and $e_{V_{0}}^{-}=1$ or $e_{V_{0}}^{+}=1$ and $e_{V_{0}}^{-}=2$. Remaining saddle type vertices are of $S^{3}$-type. 
(1)

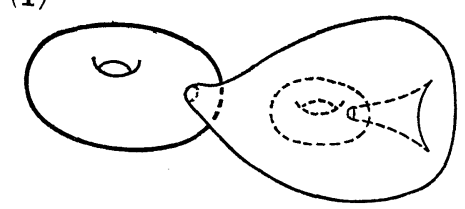

(2)

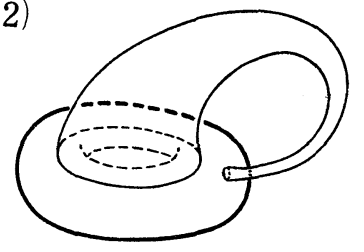

(3)

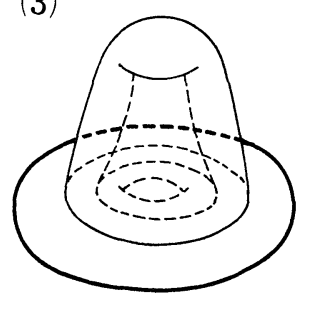

(4)

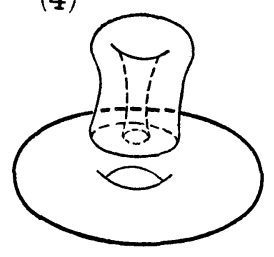

(5)

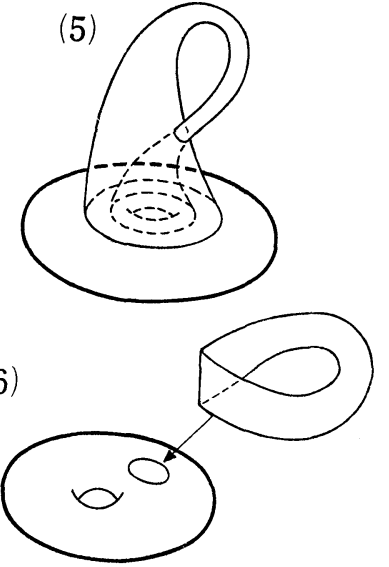

(7)

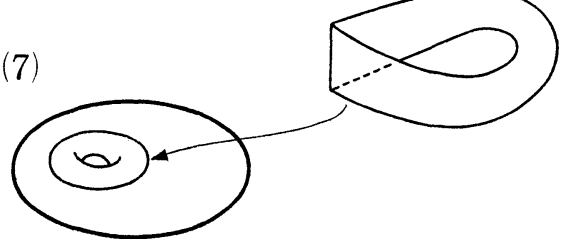

(8)

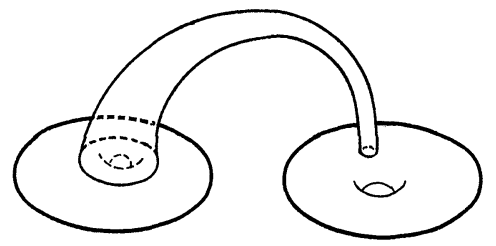

(9)

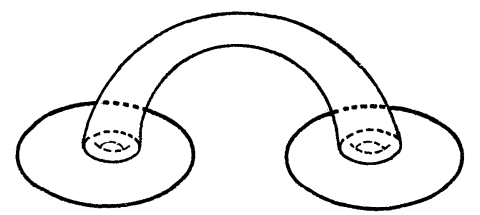

Figure (3) 
(3) (a) $M$ admits a Seifert fibration whose orbit manifold is $S^{2}$ with three exceptional fibers and (b) the associated graph $\Gamma$ has one singular vertex $V_{0}$ such that $e_{V_{0}}^{+}=e_{V_{0}}^{-}=1$ and $V_{0}$ is twisted. And any other saddle type vertex is of $S^{3}$-type.

Proof. Because $M$ is not a lens space, the Lyapunov graph must have one singular vertex (see Proposition 3). If $V_{0}$ is a vertex corresponding to a twisted closed orbit, then $e_{V_{0}}^{+}=e_{V_{0}}^{-}=1$ (see proof of Theorem 2 in [5]). We also see a topological type of basic block containing a saddle type closed orbit corresponding to the singular vertex $V_{0}$ as in Figure (3). (See [10].) It is homeomorphic to one of the followings: $S^{1} \times D^{2} \# S^{1} \times D^{2}, S^{1} \times D^{2} \# T^{2} \times I, T^{2} \times I \# T^{2} \times I$, a Seifert fibered space whose orbit space is an annulus with one exceptional fiber of index 2, (two hole disk) $\times S^{1}$, an orientable $S^{1}$-bundle over a punctured Möbius band, and $P^{3} \# T^{2} \times I$. Suppose that a basic block $B$ is homeomorphic to $T^{2} \times I \# T^{2} \times I$. Since $M$ is irreducible, we can regard $T^{2} \times I \# T^{2} \times I$ as $T^{2} \times I \# S^{3}$. And one component of a boundary torus of $T^{2} \times I$ bounds a solid torus, because $M$ has no incompressible torus. We also see that the Seifert structure of $T^{2} \times I$ is extended to this solid torus. Otherwise, $M$ is homeomorphic to $S^{3}$ or a lens space. Since a Seifert space with compressible boundary is only a solid torus (see Jaco [8]), $S^{1} \times D^{2} U$ $S^{1} \times D^{2}$ is homeomorphic to a lens space and $S^{1} \times D^{2} \cup_{T}$ (non-trivial knot-complement) is homeomorphic to $S^{3}$ by Lemma $2, M$ is a lens space or $S^{3}$. Thus $T^{2} \times I \# T^{2} \times I$ and $T^{2} \times I \# S^{1} \times D^{2}$ are excluded. Since $M$ is irreducible, we also see that $S^{1} \times D^{2} \# S^{1} \times D^{2}$ and $P^{3} \# T^{2} \times I$ are excluded. If a basic block $B$ is homeomorphic to (two hole disk) $\times S^{1}$, one component of boundary tori of this basic block B bounds a solid torus. Otherwise, $M$ admits an incompressible torus. If $\{x\} \times S^{1}$ in boundary of $B$ bounds a disk in this solid torus, then $M$ is homeomorphic to a lens space. Thus, the Seifert structure of (two hole disk) $\times S^{1}$ extends to this solid torus. Thus, it yields that each of the boundary components of (two hole disk) $\times S^{1}$ bounds a solid torus. Otherwise, $M$ is homeomorphic to $S^{3}$ or a lens space. Then we see that a Seifert space whose orbit manifold is $S^{2}$ with three exceptional fibers can be constructed from the basic block (two hole disk) $\times S^{1}$. Also a singular vertex type of $V_{0}$ which corresponds to the closed orbit of (two hole disk) $\times S^{1}$ is $e_{V_{0}}^{+}=2$ and $e_{V_{0}}^{-}=1$ or $e_{V_{0}}^{+}=1$ and $e_{V_{0}}^{-}=2$, where $V_{0}$ is untwisted. Each closed orbit corresponding to the remaining saddle type vertices is contained in 
a solid torus. They are not singular vertices by Lemma 3. Clearly, they satisfy condition (2) of the case of the lens space in Theorem B. They are $S^{3}$-type vertices. Then we see that it satisfies conditions of case (2). When a basic block is either an orientable $S^{1}$-bundle over a punctured Möbius band or a Seifert space whose orbit manifold is an annulus with one exceptional fiber of index 2 , we refer to the assertions in the proof of Theorem 1 in [10], and the proof of Theorem 4 in [10]. Then we see that the Seifert structure of these basic blocks are extended to the outside of these. Thus in the first case, $M$ has a Seifert structure whose orbit space is $P^{2}$ with at most one exceptional fiber. Also, we see that the type of the singular vertex $V_{0}$ is $e_{V_{0}}^{+}=e_{V_{0}}^{-}=1$, where $V_{0}$ is untwisted. Since remaining saddle orbits are contained in solid tori (see assertions in Theorem 1 of [10]), the remaining vertices are of $S^{3}$-type by Lemma 3. It satisfies the condition (1). In the last case, $M$ has a Seifert structure whose orbit space is $S^{2}$ with three exceptional fibers, and the basic block yields singular vertex whose vertex type is $e_{V_{0}}^{+}=e_{V_{0}}=1$, where the vertex $V_{0}$ is twisted. The remaining vertices are of $S^{3}$-type. Thus it satisfies the condition (3).

Proof of Theorem B.

First suppose $M$ is a lens space.

Necessity. As a neighbourhood of an attractor or a repeller is a solid torus, and as the graph is a tree by Proposition 1, the condition (1) is satisfied. Also, if $V$ is a closed orbit, then $k_{V}=1$. Thus $e_{V}^{+}=1$ or 2 and $e_{V}^{-}=1$ or 2 can be obtained. If $V$ is twisted, then $e_{v}^{+}=e_{v}^{-}=1$ (see the proof of Theorem 2 in [6]).

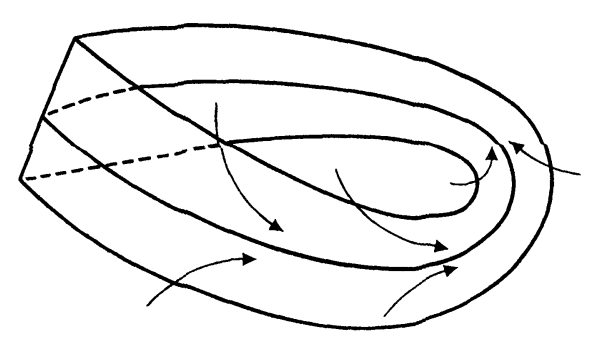

Figure (4)

Sufficiency. By using (1), (2) and (3) in Figure (3) and Figure (4), it is easy to construct an NMS flow on $S^{3}$ which has a basic block corre- 
(1)

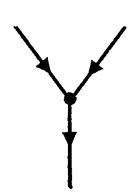

(2)

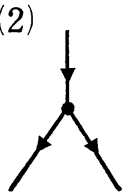

(3)

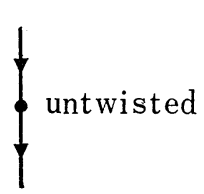

(4)

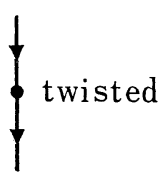

(5)

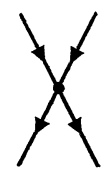

Figure (5)

sponding with the vertex type (1), (2), (3), (4) and (5) in Figure (5). Each boundary component bounds a solid torus in the both side in $S^{3}$. Therefore, by using a similar method in Theorem A, we can construct an NMS flow associated to a Lyapunov graph $\Gamma$ on any lens space. Suppose, $M$ is not a lens space.

Necessity. Since a Seifert structure of a manifold is not always unique (see Jaco [8]), under the assumption the following three possibilities occur by Proposition 4:

(1) $M$ has a structure of a Seifert manifold whose orbit space is $S^{2}$ with three exceptional fibers and also has a structure of a Seifert manifold whose orbit space is $P^{2}$ with at most one exceptional fiber.

(2) $M$ has a structure of a Seifert manifold whose orbit space is $S^{2}$ with three exceptional fibers but does not have a structure of a Seifert manifold whose orbit space is $P^{2}$ with at most one exceptional fiber.

(3) $M$ has a structure of a Seifert manifold whose orbit space is $P^{2}$ with at most one exceptional fiber but does not admit a structure of a Seifert manifold whose orbit space is $S^{2}$ with three exceptional fibers.

For example, a prism manifold corresponds to case (1), and if a Seifert fibered manifold whose orbit space is $S^{2}$ with three exceptional fibers has infinite fundamental group, then it can not have a Seifert structure whose orbit space is $P^{2}$ with at most one exceptional fiber (see Jaco [8]). Then such a manifold satisfies case (2). And under our assumptions, the fact that there is no manifold which satisfies the condi- 
tion (3) is known (see Jaco [8]). The rest of our proof is a direct consequence from Proposition 4

Sufficiency. For a construction of a basic block containing a closed orbit corresponding with a singular vertex, we can use a basic block in Figure (3). Since we can adopt a standard solid torus as the outside of the basic block, we can reduce the construction of the remaining basic blocks to the case of a lens space.

\section{§5. Special Lyapunov graphs}

By Theorem 2 in Kim [9], we see that a lens space which contains a Klein bottle can be constructed from a basic block, an orientable $S^{1}$ bundle over a punctured Möbius band. Because a solid torus or a knot complement $S^{3}-N(h)$ can not contain a Klein bottle, we see that the vertex corresponding with a closed orbit of the above basic block is a singular vertex.

On the other hand, in Theorem B we can construct any associated graph on a lens space without using a basic block which is an orientable $S^{1}$-bundle over a punctured Möbius band. This means that we can not find a singular vertex of a Lyapunov graph of an NMS flow on lens space by just looking at the ordinary Lyapunov graph. Therefore, we define a special Lyapunov graph which can distinguish a singular vertex from other vertices, by putting a mark “*” on the singular vertex.

DeFinition 10. If a special Lyapunov graph has no singular vertex and satisfies the conditions (1) and (2) in the case of the lens space of Theorem B, or conditions (0), (1) and (2) of Theorem A, we call this graph a Lyapunov graph of $S^{3}$-type. We will provide the following propositions about a special Lyapunov graph.

Proposition 5. Suppose that $\phi_{t}$ is an NMS flow on a lens space $M$ which does not contain a Klein bottle and suppose that $M$ is not a projective space $P^{3}$. Let $g$ be a Lyapunov function. Then the special Lyapunov graph $\Gamma$ associated with the flow $\phi_{t}$ and the function $g$ does not have singular vertices. In other words, the graph $\Gamma$ is of $S^{3}$-type.

Proof. Suppose that the associated Lyapunov graph $\Gamma$ has a singular vertex $V_{0}$. Since we consider a Lyapunov graph on a lens space which does not contain a Klein bottle and is not homeomorphic to $P^{3}$, a basic block which has one closed orbit corresponding to $V_{0}$ is homeo- 
morphic to one of the followings; $S^{1} \times D^{2} \# S^{1} \times D^{2}, S^{1} \times D^{2} \# T^{2} \times I$, $T^{2} \times I \# T^{2} \times I$, (two hole disk) $\times S^{1}$ and a Seifert manifold whose orbit space is an annulus with one exceptional fiber of index 2. Suppose that such a basic block is (two hole disk) $\times S^{1}$. Each connected component of $M-$ (two hole disk) $\times S^{1}$ is a solid torus as in Theorem B. Then if a simple closed curve $\{x\} \times S^{1}$ of (two hole disk) $\times S^{1} \cap$ (solid torus) bounds a disk in this solid torus, the closed orbit which corresponds to the singular vertex $V_{0}$ is included in a solid torus. By Lemma 3, it is not a singular vertex. This is a contradiction. Thus the Seifert structure of (two hole disk) $\times S^{1}$ must extend to the outside of this basic block. Here, if a lens space has a Seifert structure whose orbit manifold is $S^{2}$, then the number of exceptional fibers is at most two. Thus (two hole disk) $\times S^{1}$ is excluded. For the remaining case, using the assertions in the proof of Theorem 1 in [10], we can show that a closed orbit which corresponds to a singular vertex $V_{0}$ is also contained in a solid torus. It is a contradiction. It means that there is no singular vertex in a Lyapunov graph associated with the $\phi_{t}$ and the Lyapunov function $g$ on such a lens space.

Corollary 1. Suppose that $\phi_{t}$ is an NS flow with a Lyapunov function $g$ on a lens space which does not contain a Klein bottle and is not a projective space $P^{3}$. Let $\Gamma$ be the special Lyapunov graph associated with the flow $\phi_{t}$ and the Lyapunov function $g$. If $\Gamma$ has a vertex labelled with a saddle type closed orbit, then the vertex can not be a singular vertex.

This corollary is shown by the above proof.

Proposition 6. Suppose that $\phi_{t}$ is an NMS flow on $L(4 p, 2 p-1)$ or $P^{3}$ with a Lyapunov function $g$. Then the special Lyapunov graph $\Gamma$ associated with the flow $\phi_{t}$ and the function $g$ satisfies followings:

(1) A special Lyapunov graph on $L(4 p, 2 p-1)$ associated with a flow $\phi_{t}$ and a function $g$ is a graph of $S^{3}$-type or a graph which satisfies the followings; there is one singular vertex $V_{0}$ satisfying $e_{V_{0}}^{+}=e_{V_{0}}^{-}=1$, and it is untwisted. The remaining vertices are of $S^{3}$-type.

(2) Special Lyapunov graph $\Gamma$ on $P^{3}$ associated with a flow $\phi_{t}$ and $a$ function $g$ is a graph of $S^{3}$-type or a graph which satisfies the followings; there is one singular vertex $V_{0}$ satisfying $e_{V_{0}}^{+}=e_{V_{0}}^{-}=1$ and it is twisted. The remaining vertices are of $S^{3}$-type. 
Proof. If a flow $\phi_{t}$ on $L(4 p, 2 p-1)$ or $P^{3}$ has no closed orbit of saddle type, the graph associated with a flow $\phi_{t}$ and Lyapunov function $g$ must consist of only one sink vertex and one source vertex. It means that the graph is of $S^{3}$-type. Thus we may suppose that a flow $\phi_{t}$ on $L(4 p, 2 p-1)$ or $P^{3}$ has at least one closed orbit of saddle type. Let $V_{0}$ be a vertex which corresponds to a saddle orbit, and also we assume that the vertex $V_{0}$ is a singular vertex. If there is no such a vertex, the graph $\Gamma$ is of $S^{3}$ type. Thus if we cut $\Gamma$ at any cut point $C_{V_{0}}$ on any edge of $V_{V_{0}}$, a component of $\Gamma-\left\{C_{V_{0}}\right\}$ which does not contain $V_{0}$ corresponds to a submanifold of $M$ which is homeomorphic to a solid torus or a knot-complement $S^{3}-N(h)$, where the boundary of a compressing disk of $\partial\left(S^{3}-N(h)\right)$ is a meridian of this knot $h$.

Case (1). Suppose that a basic block which contains a closed orbit of saddle type corresponding to the singular vertex $V_{0}$ is one of followings; $S^{1} \times D^{2} \# S^{1} \times D^{2}, S^{1} \times D^{2} \# T^{2} \times I, T^{2} \times I \# T^{2} \times I$, (two hole disk) $\times S^{1}$, and a Seifert space whose orbit space is an annulus with one exceptional fiber of index 2. Then, we can show that if these basic blocks contain a closed orbit of saddle type corresponding to the vertex $V_{0}$, the closed orbit is contained in a solid torus or a knot-complement as follows. This means the vertex $V_{0}$ is not a singular vertex. Thus we can omit all basic blocks in the above cases. For example let a basic block be a Seifert space whose orbit manifold is an annulus with one exceptional fiber of index 2 . Since $L(4 p, 2 p-1)$ has no incompressible tori, one component of $L(4 p, 2 p-1)-($ the above basic block $B$ ) must be a solid torus. By the assertions 3 in the proof of Theorem 1 of [10], we see that a fiber of this basic block does not bound a disk in this solid torus. Otherwise, $L(4 p, 2 p-1)$ is homeomorphic to $P^{3}$. This means that a Seifert structure extends to this soild torus. Then another component of $L(4 p, 2 p-1)-B$ is a solid torus, and also this Seifert structure extends to this solid torus. If $L(4 p, 2 p-1)$ has a Seifert structure whose orbit space is $S^{2}$, the number of singular fibers is at most two. Then the vertex $V_{0}$ is not a singular vertex. Therefore, suppose that a basic block which contains a closed orbit of saddle type is homeomorphic to an orientable $S^{1}$-bundle over a punctured Möbius band. Since a solid torus and a non-trivial knot-complement $S^{3}-N(h)$ can not contain a Klein bottle and since the vertex $V_{0}$ corresponds a closed orbit saddle type of an orientable $S^{1}$-bundle over a punctured Möbius band, there is 
one singular vertex $V_{0}$ such that $e_{V_{0}}^{+}=e_{V_{0}}^{-}=1$ and it is untwisted. For the remaining vertices, they are contained in a solid torus or a nontrivial knot-complement by Proposition 3. Thus they are of $S^{3}$-type by Lemma 3.

Case (2). Suppose that a basic block which contains a closed orbit of saddle type corresponding to a singular vertex $V_{0}$ is one of followings; $S^{1} \times D^{2} \# S^{1} \times D^{2}, S^{1} \times D^{2} \# T^{2} \times I, T^{2} \times I \# T^{2} \times I$ and (two hole disk) $\times S^{1}$. Then we can show that the closed orbit of saddle type corresponding to the vertex $V_{0}$ is contained in a solid torus or a knotcomplement by a proof similar to case (1). This is a contradiction. Then we can omit these basic blocks. Suppose that a basic block $B$ which has a closed orbit of saddle type is a Seifert space whose orbit manifold is an annulus with one exceptional fiber of index 2 . Since $P^{3}$ has no incompressible torus, one component of $P^{3}-B$ is a solid torus. If a Seifert structure of this basic block $B$ extends to this solid torus, another component of $P^{3}-B$ must be a solid torus. Otherwise, $P^{3}$ admits an incompressible torus, or it is homeomorphic to $S^{3}$ by Lemma 2 . If a fiber of this basic block $\mathrm{B}$ bounds a disk $D^{2}$ in this solid torus, and if another component of $P^{3}-B$ is a non-trivial knot-complement $S^{3}-N(h)$, then $P^{3}$ is homeomorphic to $S^{3}$. Because the above disk $D^{2}$ is a meridian of $h, P^{3}$ is homeomorphic to (solid torus) $\cup_{T}$ (non-trivial knotcomplement). Thus $P^{3}$ is homeomorphic to $S^{3}$ by Lemma 2. Then each component of $P^{3}-B$ is a solid torus. Using the proof of Theorem 2 in [10], we see that a closed orbit saddle type of the above basic block is contained in a solid torus. Then, in this case, the vertex $V_{0}$ is not a singular vertex. This is a contradiction. Thus, this basic block is also omitted. A solid torus and a knot-complement $S^{3}-N(h)$ can not contain a projective plane $P^{2}$. Therefore, if such a basic block is homeomorphic to $P^{3} \# T^{2} \times I$, the vertex $V_{0}$ corresponding to the closed orbit of saddle type of $P^{3} \# T^{2} \times I$ is a singular vertex. This vertex type is $e_{V_{0}}^{+}=e_{\bar{V}_{0}}=1$, where $V_{0}$ is twisted. Another vertex is of $S^{3}$-type, because another closed orbit is contained in a solid torus or a knot-complement. Then, Proposition 6 is proved.

Remark 9. For the case (1), since $L(4 p, 2 p-1)$ is not homeomorphic to $P^{3}$, a basic block is not homeomorphic to $P^{3} \# T^{2} \times I$, For the case (2), a basic block is not homeomorphic to an orientable $S^{1}$-bundle over a punctured Möbius band, since $P^{3}$ does not contain a Klein bottle. 


\section{§ 6. Examples of special Lyapunov graphs of NS flows}

By using a construction in [5], we can show that there exist some examples concerning an NS flow which is not an NMS flow and whose associated Lyapunov graph has one singular vertex on $L(4 p, 2 p-1)$ and a Seirfert space whose orbit manifold is $S^{2}$ with three exceptional fibers.

Case (1). A Seifert space whose orbit manifold is $S^{2}$ with three exceptional fibers; A graph associated with a flow $\phi_{t}$ and a Lyapunov function $g$ has one singular vertex $V_{0}$ such that $e_{V_{0}}^{+} \leqq k_{V_{0}}+1, e_{V_{0}} \leqq k_{V_{0}}+1$ and $k_{V_{0}}+2=e_{V_{0}}^{+}+e_{V_{0}}^{-}$. Any other vertex $V$ which is neither an attractor nor a repeller satisfies the following conditions; $e_{V}^{+} \leqq k_{V}+1$, $e_{\bar{V}}^{\bar{V}} \leqq k_{V}+1$ and $e_{V}^{+}+e_{\bar{V}}^{-} \geqq k_{V}+1$.
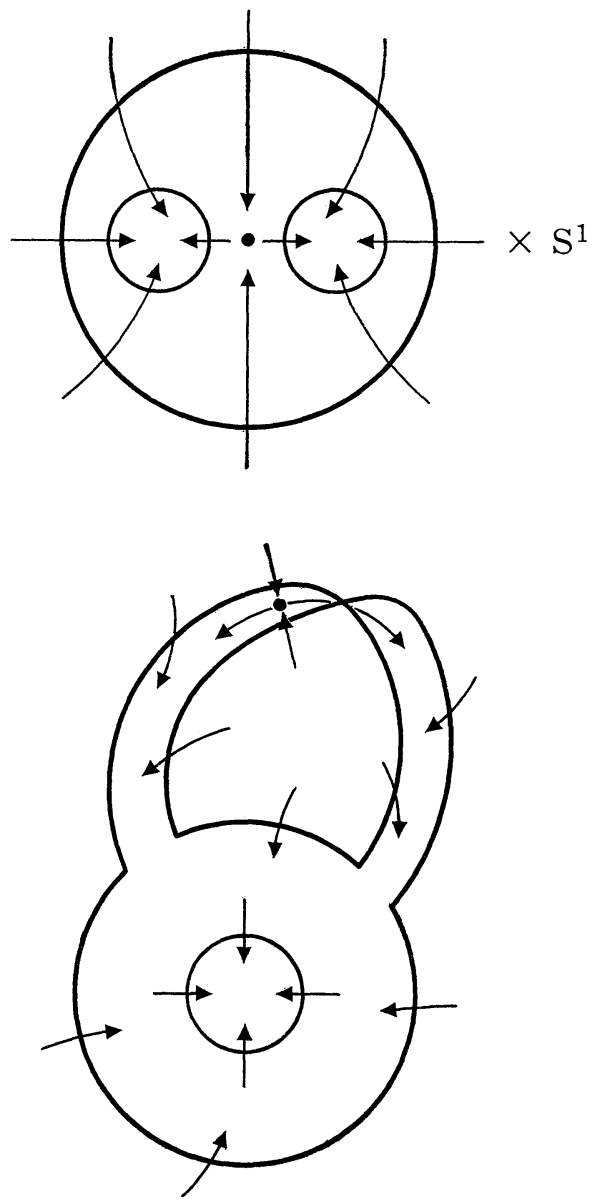

Figure (6) 
Case (2). A prism manifold and a lens space $L(4 p, 2 p-1) ; \mathrm{A}$ Lyapunov graph associated with a flow $\phi_{t}$ and a Lyapunov function $g$

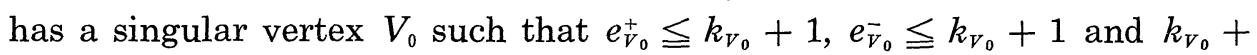
$1=e_{V_{0}}^{+}+e_{V_{0}}^{-}$, and also any other vertex $V$ which is neither attracting nor repelling satisfies the following conditions; $e_{V}^{+} \leqq k_{V}+1, e_{V}^{-} \leqq k_{V}+1$ and $k_{V}+1 \leqq e_{V}^{+}+e_{V}^{-}$. For our construction of examples of case (1) (resp. case (2)) we use a two hole disk (resp. a punctured Möbius band) on a basic block (two hole disk) $\times S^{1}$ (resp. an orientable $S^{1}$-bundle over a punctured Möbius band) as a cross section of the flow $\phi_{t}$ first return map has one saddle point (see Figure (6)). Next, we add new fixed points on this cross section as follows.

Case (1). We add $\left(k_{V_{0}}-e_{V_{0}}^{+}\right)$pairs of a sink and a saddle, and also add $\left(k_{V_{0}}+1-e_{V_{0}}^{-}\right)$pairs of a source and a saddle. Finally we add $\left(n-k_{V_{0}}\right)$ nilpotent handles.

Case (2). We add $\left(k_{V_{0}}-e_{V_{0}}^{+}\right)$pairs of a sink and a saddle, and also add $\left(k_{V_{0}}-e_{V_{0}}^{-}\right)$pairs of a source and a saddle. Finally we add $\left(n-k_{V_{0}}\right)$ nilpotent handles.

Then a basic block which contains a basic set corresponding to a singular vertex $V_{0}$ in case (1) and case (2) is obtained by a method similar to the proof of Theorem 1 of [6]. Now, we will construct a fitted NS flow whose Lyapunov graph satisfies case (1) on a Seifert fibered manifold $M$ whose orbit space is $S^{2}$ with three exceptional fibers. For case (2), we can construct a fitted NS flow similary. Let $B$ be a basic block which has a basic set corresponding to a singular vertex $V_{0}$. And let $X_{1}^{i}, X_{2}^{i}, \cdots, X_{N_{i}}^{i}$ be basic blocks contained in $M-B\left(1 \leqq i \leqq k_{V_{0}}+2\right)$. Because we can regard each component of $M-B$ as a standard solid torus, we can adopt basic block as in Theorem 1 of [6] for $X_{1}^{i}, X_{2}^{i}, \cdots, X_{N_{i}}^{i}$ $\left(1 \leqq i \leqq k_{V_{0}}+2\right)$. Thus they can be embedded in $S^{3}$ and their boundary components are all standard tori. Then by choosing attaching maps $g_{1}^{i}, g_{2}^{i}, \cdots, g_{N_{i-1}}^{i}\left(1 \leqq i \leqq k_{V_{0}}+2\right)$, we can construct $X_{1}^{i} \cup_{g_{1}^{i}} X_{2}^{i} \cup_{g_{2}^{i}} \cdots$ $\bigcup_{g_{N_{i}-1}^{i}} X_{N_{i}}^{i}$ as a standard solid torus $\left(1 \leqq i \leqq k_{V_{0}}+2\right)$. Next, choosing attaching maps $h_{1}, h_{2}, \cdots, h_{k_{V_{0}+2}}$, we glue these basic blocks $X_{1}^{i} \cup_{g_{1}^{i}} X_{2}^{i} \cup_{g_{2}^{i}} \cdots$ $\bigcup_{g_{N_{i}-1}^{i}} X_{N_{i}}^{i}\left(1 \leqq i \leqq k_{V_{0}}+2\right)$ along the boundaries of the basic block $B$. Then we can construct a non-singular flow $\phi_{t}$ on a Seifert fibered manifold whose orbit space is $S^{2}$ with three exceptional fibers. By changing each $g_{j}^{i}\left(1 \leqq j \leqq N_{i}-1\right)\left(1 \leqq i \leqq k_{V_{0}}+2\right)$, into $g_{j}^{i^{\prime}}$ which is isotopic to $g_{j}^{i}$, and 
also changing each $h_{s}$ into $h_{s}^{\prime}\left(1 \leqq s \leqq k_{V_{0}}+2\right)$ which is isotopic to $h_{s}$, we can reconstruct a fitted flow $\phi_{t}^{\prime}$ from the flow $\phi_{t}$ by Proposition 2. Let $X_{K_{i}}^{i}\left(1 \leqq K_{i} \leqq N_{i}\right)$ be a basic block which attaches to the basic block $B\left(1 \leqq i \leqq k_{V_{0}}+2\right)$. Since each boundary of these basic blocks is a standard torus in $S^{3}$, then we can assume that $X_{1}^{i} \cup_{g_{1}^{i}} \cdots \cup_{g_{K_{i}-2}^{i}} X_{K_{i}-1}, X_{1}^{i} \cup_{g_{1}^{i}} \cdots$ $\bigcup_{g_{K_{i}-2}^{i}} X_{K_{i}-1}^{i} \bigcup_{g_{K_{i}-1}^{i}} X_{K_{i}}^{i} \bigcup_{g_{K_{i}}^{i}} \cdots \cup_{g_{N_{i}-1}^{i}} X_{N_{i}}^{i}, X_{K_{i+1}}^{i} \bigcup_{g_{K_{i+1}}^{i}} \cdots \cup_{g_{L_{i}-1}^{i}} X_{L_{i}}$, $X_{K_{i+2}}^{i} \bigcup_{g_{K_{i}+2}^{i}} \cdots \cup_{g_{L_{i}-1}^{i}} X_{L_{i}}, \cdots$, and $X_{N_{i}}$ are solid tori, where the basic blocks $X_{L_{i}}$ and $X_{N_{i}}\left(1 \leqq k_{i} \leqq L_{i} \leqq N_{i}\right)$ contain a repelling closed orbit or an attracting closed orbit. (If necessary, we suitably change the lower indexes of basic blocks $X_{1}^{i}, X_{2}^{i}, \cdots$, and $X_{N_{i}}^{i}$ ). By using Alexander trick, we see that $B \cup_{h_{s}^{\prime}}\left(X_{1}^{s} \cup_{g_{1}^{s}} X_{2}^{s} \cup_{g_{2}^{s}} \cdots \cup_{g_{N_{s}-1}^{s}} X_{N_{s}}^{s}\right)$ is homeomorphic to $B \bigcup_{h_{s}}$ $\left(X_{1}^{s} \cup_{g_{1}^{s}} X_{2}^{s} \bigcup_{g_{2}^{s}} \cdots \cup_{g_{N_{s}-1}^{s}} X_{N_{s}}\right), \quad B \cup_{h_{s}^{\prime}}\left(X_{1}^{s} \cup_{g_{1}^{s^{\prime}}} \cdots \cup_{g_{K_{s}-2}^{s^{\prime}}} X_{K_{s}}^{s} \cup_{g_{K_{s}-1}^{s^{\prime}}} \cdots\right.$ $\left.\bigcup_{g_{N_{s}-1}^{s}} X_{N_{s}}^{s}\right)$ is homeomorphic to $B \bigcup_{h_{s}^{\prime}}\left(X_{1}^{s} \bigcup_{g_{1}^{s^{\prime}}} \cdots \cup_{g_{K_{s}-2}^{s}} X_{K_{s}-1}^{s} \bigcup_{g_{K_{s}-1}^{s}} \cdots\right.$ $\left.\bigcup_{g_{N_{s}-1}^{s}} X_{N_{s}}\right), B \bigcup_{h_{s}^{\prime}}\left(X_{1}^{s} \bigcup_{g_{1}^{s^{\prime}}} \bigcup_{g_{K_{s}-1}^{s^{\prime}}} X_{K_{s}}^{s} \bigcup_{g_{K_{s}}^{s^{\prime}}} X_{K_{s}+1}^{s} \bigcup_{g_{K_{s}+1}^{s}} \cdots \bigcup_{g_{N_{s}-1}^{s}} X_{N_{s}}\right)$ is homeomorphic to $B \bigcup_{h_{s}^{\prime}}\left(X_{1}^{s} \bigcup_{g_{1}^{s^{\prime}}} \cdots \cup_{K_{s-2}^{\prime}} X_{K_{s-1}}^{s} \bigcup_{g_{K_{s}-1}^{s^{\prime}}} X_{K_{s}}^{s} \bigcup_{g_{K_{s}}^{s}} \cdots \cup_{g_{N_{s}-1}^{s}}\right.$ $\left.X_{N_{s}}\right)$, etc. Thus we see that a homeomorphism type of $M$ does not change.

Remark 10. Of course, if a singular vertes $V_{0}$ corresponds to a closed orbit of saddle type, we can construct an NS flow which is not an NMS flow on $P^{3}, L(4 p, 2 p-1)$ and a Seifert fiberd space whose orbit manifold is $S^{2}$ with three exceptional fibers by similar construction.

Question. Does any Lyapunov graph on $L(2 p-1, q)$ associated with an NS flow $\phi_{t}$ and a Lyapunov function $g$ admit no singular vertex?

\section{REFERENCES}

[1] R. Bowen, One dimensional hyperbolic set for flows, J. Differential Equations, 12 (1972), 173-179.

[2] J. Birman and R. F. Williams, Knotted periodic orbits in dynamical system II: knot holders for fibered knots in Contemporary Mathematics, Low Dimensional Topology, Vol. 20, 1983.

[3] J. Franke and J. Selgrade, Hyperbolicity and chain recurrence, J. Differential Equations, 26 (1977), 27-36.

[4] J. Franks, Homology and Dynamical systems, C.B.M.S. Regional Conf. Series in Math., 49 Amer. Math. Soc. Providence R.I., 1982.

[ 5 ] — Symbolic dynamics in flows on three manifold, Trans. Amer. Math. Soc., 279 (1983), 231-236.

[ 6 ] — Non-singular Smale flow on $S^{3}$, Topology, 24 No. 3 (1985), 265-282.

[ 7 ] J. Hempel, 3-manifolds, Ann. of Math. Studies No. 86, Princeton University press, Princeton N.J., 1976.

[ 8 ] W. Jaco, Lectures on three manifold topology C.B.M.S. Regional conf. Series in 
Math., 43 Amer. Math. Soc. Providence, R.I., 1980.

[ 9 ] P. K. Kim, Some 3-manifold which admit Klein bottles, Trans. Amer. Math. Soc., 244 (1978), 299-312.

[10] T. Kobayashi, Primitive links of non-singular Morse-Smale flows on the special Seifert fiberd manifold, Topology Appl., 20 No. 1, (1985), 67-78.

[11] J. Morgan, Non-Singular Morse Smale flows on 3-dimensional manifold, Topology, 18 (1978), 41-53.

[12] C. Pugh and M. Shub, Embedding suspension of subshifts of finite type in $S^{3}$ in Contributions to Geometry and Analysis, C. Percelli and R. Sacksteder eds., Johns Hopkins University Press, 1981.

[13] D. Rolfsen, Knots and Links, Publish or Perish Inc., Berkeley, 1976.

[14] K. Sasano, Links of closed orbits of non-singular Morse-Smale flows, Proc. Amer. Math. Soc., 88 (1983), 727-734.

[15] S. Smale, Differentiable dynamical systems, Bull. Amer. Math. Soc., 73 (1967), 797-817.

[16] M. Wada, Links which consist of the closed orbits of non-singular Morse-Smale flow on 3-sphere, preprint.

\author{
Department of Mathematics \\ School of Science \\ Nagoya University \\ Chikusa-ku, Nagoya 464 \\ Japan
}

